\title{
Use of medetomidine, midazolam, ketamine and sevoflurane as an anesthetic protocol for domestic chickens
}

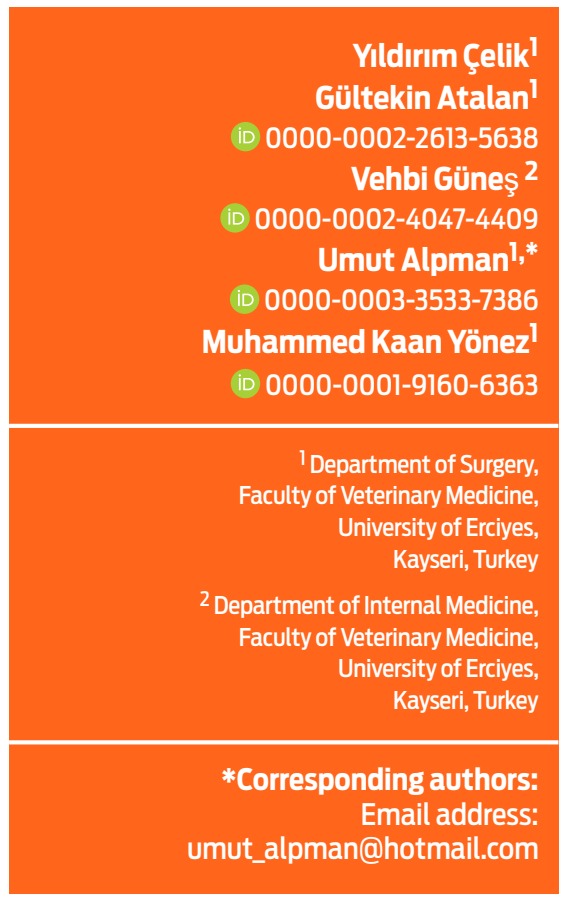

Accepted: $2019-09-25$

Published: 2020-03-31

Additional information and declarations can be found on page 10

@ Copyright 2020 Yildirım Çeliket al.

open access $\boldsymbol{\mathcal { O }}$

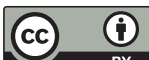

Distributed under Creative Commons CC-BY 4.0

\begin{abstract}
Changes in physiological and biochemical parameters after administration of medetomidine (MED), midazolam (MID), ketamine (KET) and a 2\% of the inhalation anesthetic sevoflurane (SEVO), were investigated in domestic chickens. The anesthetic protocol began with a simultaneous intrapectoral injection (IP) of MED (50 $\mu \mathrm{g} / \mathrm{kg})$ and MID $(0.5 \mathrm{mg} / \mathrm{kg})$, followed by IP administration of $25 \mathrm{mg} / \mathrm{kg}$ of KET 10 min later. Anesthesia was then maintained for $30 \mathrm{~min}$ by $2 \%$ SEVO (with a $500 \mathrm{ml} / \mathrm{min}$ oxygen flow), using an Ayres $T$ piece device. Heart and respiratory rates, cloacal temperature, reflex response and electrocardiogram (ECG) parameters were recorded at time zero (TO) before anesthesia (BA, baseline values), at time of MED + MID administration (T1), at time of ketamine injection (T2), 30 min after the start of SEVO inhalation (T3) and at recovery. Blood was also drawn at TO and T3 to assess albumin, creatinine, glucose and liver enzyme concentrations. Cloacal temperature, heart and respiratory rates differed from baseline values at all time intervals during anesthesia $(p<0.05)$. Heart rate decreased following the MED + MID injection (at T1, T2 and T3), and partially recovered by the reanimation period. Reflex response also differed between time 0 and all anesthesia time points $(p<0.05)$. Mean amplitude of the $P$ wave of the ECG was increased during MED + MID (T1) and KET (T2) anesthesia. The mean ST interval showed a large increase at $\mathrm{T} 1$, which was maintained throughout anesthesia $(p<0.05)$. Albumin, glucose and the ALT enzyme decreased between TO and T3. In conclusion, the use of MED + MID + KET and SEVO
\end{abstract}

\section{Cite this as:}

Çelik Y, Atalan G, Güneş V, Alpman U, Yönez MK. Use of medetomidine, midazolam, ketamine and sevoflurane as an anesthetic protocol for domestic chickens. Veterinaria México OA. 2020;7(1). doi: 10.22201/fmvz.24486760e.2020.1.620. 


\section{Original Research}

Dol: http://dx.doi.org/10.22201/fmvz.24486760e.2020.1.620

Vol. 7 I No. 1 I January-March I 2020

as an anesthetic combination altered cardiorespiratory and biochemical parameters of chickens, but no life-threatening effects were observed as a result of these changes. Hence, this drug combination can be adequately used as an anesthesia protocol in chickens.

Keywords: Anesthesia; Chicken; Preanesthesia 


\section{Introduction}

Anesthesia has critical importance in avian medicine and surgery. In poultry, medetomidine, xylazine, midazolam, diazepam, butarphanol, atropine and glycoprolate are commonly used as pre-anesthetics drugs. ${ }^{1}$ Medetomidine is a potent and selective $\alpha$-2 adrenoreceptor agonist, but it is also one of the safest premedication agent used in birds. ${ }^{2}$ When applied by intramuscular injection $(25 \mu \mathrm{g} / \mathrm{kg})$, it causes sedation and loss of reflexes in 8-day-old chicks. ${ }^{3}$ Ketamine, an injectable cyclohexane, is a dissociative drug that produces poor muscle relaxation in animals, but that has been deemed suitable as an anesthetic agent for birds. 4,5 However, it can only be used on its own for short procedures, since it can cause muscle tremors, catalepsy (muscle stiffness), myotonic contractions, opisthotonus and rough recovery periods. ${ }^{5,6}$ Midazolam has a relatively rapid metabolic clearance compared to the other benzodiazepines and hence has a short duration of action in all species. It has anticonvulsant, anxiolytic, sleep-inducing, muscle relaxing, and sedating effects when administered orally, intramuscularly, or intravenously. ${ }^{7,8}$ When combined with ketamine, a quick onset and deep anesthesia, with good muscle relaxation and a reduced recovery time was attained in broiler chicks. ${ }^{9}$ Isoflurane and sevoflurane are commonly used inhaled agents that are also used in birds for induction maintenance of anesthesia. ${ }^{10}$ Sevoflurane has a lower blood/gas solubility coefficient than isoflurane. It also has shorter recovery times compared to other anesthetic drugs in long-term anesthesia. ${ }^{11}$ It does not affect heart rate in chickens, and results in less apnea and irritation when administered with a mask. ${ }^{12}$

Surgical procedures are frequently performed in poultry, and an adequate combination of anesthetics is valuable for clinical practice. In this study, we examined the effects of a combination of medetomidine, midazolam, ketamine and sevoflurane anesthesia on physiological and biochemical parameters in chickens.

\section{Materials and methods} Animals

Twenty, 72-day old healthy Lohmann Brown chicks, with a body weight of $1.7 \pm 0.3 \mathrm{~kg}$ were used for this study. To reduce handling stress, all daily management procedures, as well as sampling and testing protocols (blood collection, injections, and electrocardiography) were performed by the same person.

This study was approved by the Erciyes University local Ethics Committee and complied with the International Guidelines for the use of animals in Biomedical Research.

\section{Anesthesia preparation}

There were no feeding restrictions for animals before anesthesia. The dose of the anesthetic agent was calculated for each individual based on its weight. Chicks were left alone to provide for a relaxing environment for 10 minutes before applying the pre-anesthetic agent. Baseline values were taken immediately before anesthesia (time point 0 / TO) and recorded in an examination form. 


\section{Anesthesia protocol}

A combination of midazolam (MID) $(0.5 \mathrm{mg} / \mathrm{kg}$, Zolamide $5 \mathrm{ml} / 5 \mathrm{mg} 5 \mathrm{amp}$, Defarma, Turkey) and medetomidine (MED) $(50 \mu \mathrm{g} / \mathrm{kg}$, Domitor $10 \mathrm{ml}$, Pfizer, Finland) was injected into the pectoral muscles of each chicken mixed with $1 \mathrm{ml}$ of saline solution $(0.9 \% \mathrm{NaCl})$ as preanesthetic [time point $1(\mathrm{~T} 1)$ ]. Ten minutes later a $25 \mathrm{mg} / \mathrm{kg}$ (Ketasol 10\%, $10 \mathrm{ml}$, Interhas, Turkey) of ketamine-HCl (KET) was IP applied [time point 2 (T2)]. After a further 10 minutes, chicks were intubated with silicone tubes (2-3 $\mathrm{mm}$ in diameter) to maintain anesthesia for 30 min using 2\% sevofluorane (SEVO) (Sevorane Liquid 100\%, 250 cc, Abbvie, England) with 500 $\mathrm{ml} / \mathrm{min}$ oxygen flow [time point 3 at end of these 30 minutes (T3)]. An SMS 2000 Classic inhalation anesthesia device applying the modified Jackson-Rees (Ayres T piece) system was used.

\section{Electrocardiographic examination}

Electrocardiographic findings were recorded (CAREWELL $1103 \mathrm{G}$ Veterinary ECG Device) at T0 before anesthesia and at time points T1, T2 and T3 during anesthesia. Bipolar extremity (I, II, III) and increased unipolar (aVR, aVL, aVF) limb leads were used for electrocardiograms with the aid of crocodile electrodes. ECG parameters and intervals were recorded at derivation II, at a $25 \mathrm{~mm} / \mathrm{s}$ speed.

\section{Clinical examination and reflex response assessment}

Changes in palpebral and pedal reflexes, as well as head, neck, wing and body position alterations were recorded for all anesthesia time points. Also, cloacal body temperature $\left({ }^{\circ} \mathrm{C}\right)$ and heart-respiratory rates were concurrently recorded.

Reflex responses were graded according to the following criteria, as has been previously reported: ${ }^{13}$

1. Individual is alive, responsive and alert.

2. Very little or partial response to stimulus.

3. No response to stimulation, deep anesthetic effect.

\section{Biochemical analyses}

Blood was drawn $(3 \mathrm{ml})$ from the vena brachialis of each individual into tubes without anticoagulant at TO (baseline) and at T3 for biochemical analyses. Blood was centrifuged at $1500 \mathrm{rpm}$ for 15 minutes to obtain sera. Concentrations of alanine aminotransferase (ALT), aspartate aminotransferase (AST), albumin (ALB), creatinine (CR), and glucose (GLU), as well as total protein and LDH were assessed.

\section{Statistical analyses}

The data was evaluated with the statistical package program IBM SPSS Statistics 22.0 (IBM Corp., Armonk, New York, USA). Values are reported as mean \pm standard deviation (SD) and median (min-max) values. Normal distributions of data were evaluated by the Shapiro Wilk normality test and Q-Q graphs. Paired $t$ and 
Table 1. Physiological parameters before, during and after anesthesia (mean \pm SD)

\begin{tabular}{|l|c|c|c|c|c|}
\hline \multicolumn{1}{|c|}{ Parameters } & T0 & T1 & T2 & T3 & Reanimation \\
\hline Cloacal temperature $\left({ }^{\circ} \mathrm{C}\right)$ & $41.27 \pm 0.28^{\mathrm{a}}$ & $40.91 \pm 0.51^{\mathrm{b}}$ & $40.33 \pm 0.45^{\mathrm{b}}$ & $39.17 \pm 0.80^{\mathrm{c}}$ & $40.13 \pm 0.65^{\mathrm{b}}$ \\
\hline Heart rate & $242.70 \pm 37,6^{\mathrm{a}}$ & $204.90 \pm 27.16^{\mathrm{b}}$ & $203.10 \pm 37.83^{\mathrm{b}}$ & $194.60 \pm 39.68^{\mathrm{c}}$ & $208.73 \pm 36.40^{\mathrm{b}}$ \\
\hline Respiratory rate & $43.80 \pm 7.73^{\mathrm{a}}$ & $22.80 \pm 6.87^{\mathrm{b}}$ & $21.40 \pm 9,64^{\mathrm{b}}$ & $16.10 \pm 5.74^{\mathrm{c}}$ & $24 \pm 6.44^{\mathrm{b}}$ \\
\hline
\end{tabular}

$a, b, c$ Different letters shows statistically significantly difference in the same line $(p<0.05)$.

SD: standard deviation; TO: before anesthesia; T1: 10 minutes after injection of medetomidine-midazolam; T2: 10 minutes after injection of ketamine; T3: 30 minutes after administration of sevoflurane.

Wilcoxon test were used when two consecutive measurements were assessed. Analysis of variance for repeated measures and the Friedman test were used when four successive values were assessed. In case of a difference, parametric and nonparametric Student-Newman-Keuls multiple comparison test was used. Statistical differences were set at $p<0.05$.

\section{Results}

\section{Physiological parameters}

Mean cloacal body temperature decreased and remained low throughout anesthesia $(T 1, T 2, T 3$ and recovery vs T0; $p<0.05)$. Respiratory rate was lower at $T 1$, T2 and T3, when compared to T0 $(p<0.05)$ but partially rose during recovery time. Similarly, heart rate decreased gradually during anesthesia $(p<0.05)$ but increased during reanimation. Mean values of physiological parameters are shown in Table 1.

\section{Electrocardiographic examination}

Tachycardia was observed in all chickens during the preanesthetic period. Sinus tachycardia and ventricular tachycardia were the most common findings observed during general anesthesia in ECG records. Mean amplitude of the $P$ wave increased at T1 and T2 ( $P$ 0.05), but partially decreased by T3 (Table 2). As for mean PR time values, there was an increase at $\mathrm{T} 1$ that returned to the baseline values by $\mathrm{T} 2$ and remained unchanged thereafter. There were no differences for the QRS time interval. The mean ST interval increased at T1 and remained higher than baseline values at T2 and T3 ( $p<0.05)$. The QT and T wave amplitude values did not differ from TO at any time during anesthesia. Mean values for ECG parameters are presented in Table 2. Examples of ECG prints during anesthesia are shown in Figure 1.

\section{Clinical findings}

Reflexes were lessened from anesthesia onset ( $\mathrm{T} 1)$ and later lost, to be recovered by the reanimation period in all individuals $(p<0.05)$. The palpebral reflex was still present in 4 of the 20 chicks at $\mathrm{T} 1$ but had already lessened in the rest of the 
Table 2. ECG parameters before, during and after anesthesia mean \pm SD

\begin{tabular}{|c|c|c|c|c|}
\hline ECG Parameters & T0 & T1 & T2 & T3 \\
\hline P amp (mv) & $0.02 \pm 0.11^{\mathrm{a}}$ & $0.15 \pm 0.04^{\mathrm{b}}$ & $0.78 \pm 0,28^{\mathrm{b}}$ & $0.4 \pm 0.05^{\mathrm{c}}$ \\
\hline P time (s) & $0.03 \pm 0.18$ & $0.04 \pm 0.07$ & $0.02 \pm 0.0$ & $0.02 \pm 0.00$ \\
\hline PR time (s) & $0.06 \pm 0.07$ & $0.09 \pm 0.16$ & $0.07 \pm 0.08$ & $0.06 \pm 0.01$ \\
\hline QRS time (s) & $0.06 \pm 0.09$ & $0.03 \pm 0.00$ & $0.03 \pm 0.0$ & $0.04 \pm 0.01$ \\
\hline ST time (s) & $0.04 \pm 0.03^{\mathrm{a}}$ & $0.08 \pm 0.01^{\mathrm{b}}$ & $0.09 \pm 0.01^{\mathrm{b}}$ & $0.13 \pm 0.17^{\mathrm{b}}$ \\
\hline QT time (s) & $0.11 \pm 0.08$ & $0.12 \pm 0.01$ & $0.12 \pm 0.02$ & $0.13 \pm 0.01$ \\
\hline T amp (mv) & $0.19 \pm 0.16$ & $0.25 \pm 0.12$ & $0.21 \pm 0.09$ & $0.22 \pm 0.09$ \\
\hline
\end{tabular}

$a, b, c$ Different superscripts within the same row indicate statistical differences $(p<0.05)$.

amp: amplitude; ECG: electrocardiogram; SD: standard deviation; TO: before anesthesia; T1: 10 minutes after injection of medetomidine-midazolam; T2: 10 minutes after injection of ketamine; T3: 30 minutes after administration of sevoflurane.
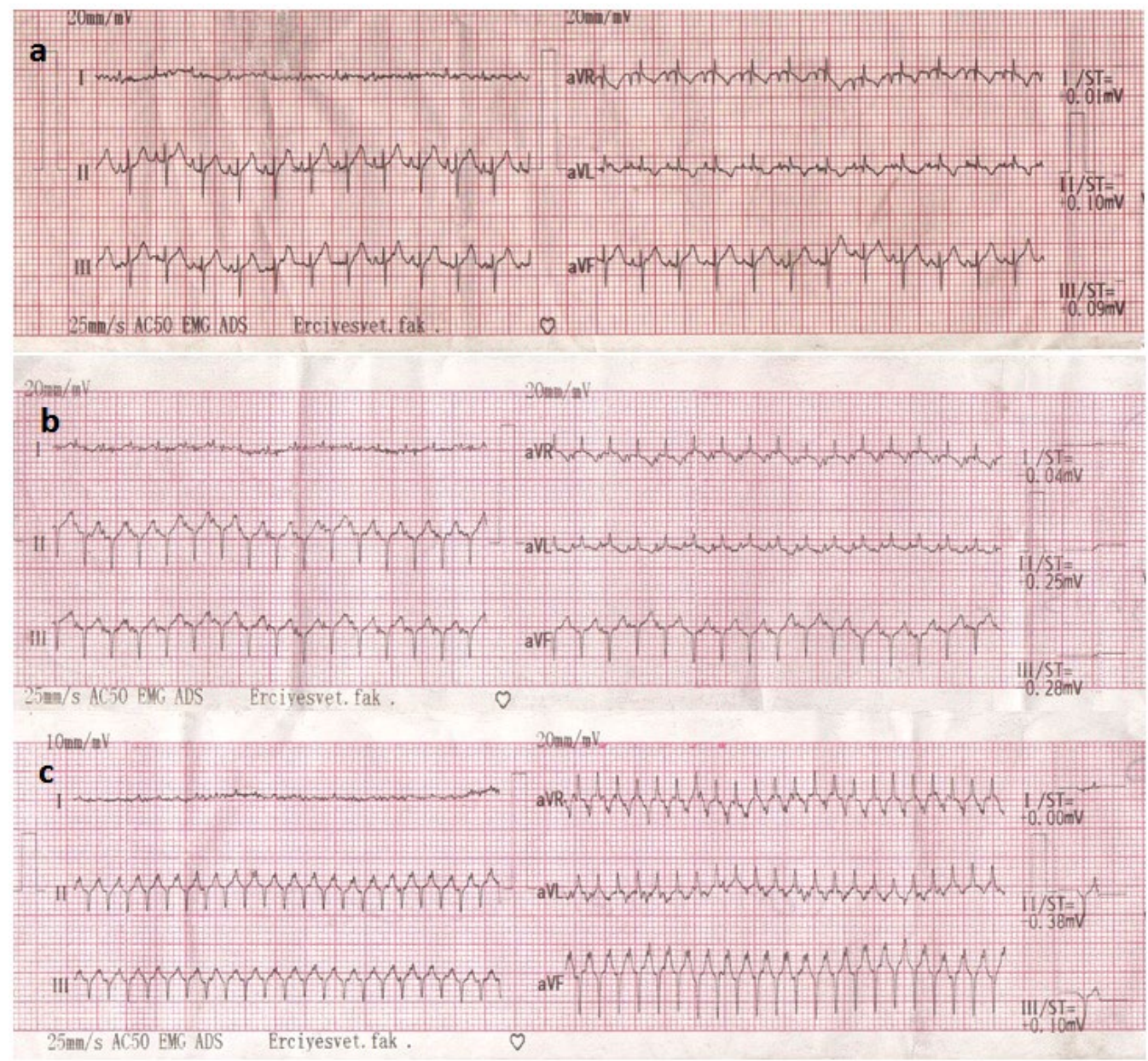

Figure 1. Examples of electrocardiogram prints showing different parameter results from anesthetized chicks. a) Normal sinus rhythm after medetomidine and midazolam administration, ST segment elevations. b) Sinus tachycardia after ketamine administration. c) Ventricular tachycardia after sevoflurane administration. 
Table 3. Reflex response grading at different time points during the anesthetic protocol (median)

\begin{tabular}{|l|c|c|c|c|c|}
\hline \multicolumn{1}{|c|}{ Clinical reflex } & T0 & T1 & T2 & T3 & Recovery \\
\hline Wing withdrawal & $1^{\mathrm{a}}$ & $2^{\mathrm{a}}$ & $3^{\mathrm{a}}$ & $3^{\mathrm{a}}$ & 1 \\
\hline Palpebral reflex & $1^{\mathrm{a}}$ & $2^{\mathrm{a}}$ & $3^{\mathrm{a}}$ & $3^{\mathrm{a}}$ & 1 \\
\hline Pedal reflex & $1^{\mathrm{a}}$ & $2^{\mathrm{a}}$ & $3^{\mathrm{a}}$ & $3^{\mathrm{a}}$ & 1 \\
\hline
\end{tabular}

a Indicates differences from TO within the same row $(p<0.05)$.

TO: before anesthesia; T1: 10 minutes after injection of medetomidine-midazolam; T2: 10 minutes after injection of ketamine; T3: 30 minutes after administration of sevoflurane.

Table 4. Biochemical parameters of anesthetized chicks at T0 and T3 mean \pm SD

\begin{tabular}{|l|c|c|}
\hline Biochemical parameters & TO & T3 \\
\hline Albumin (g/dl) & $2.05 \pm 0.41^{\mathrm{a}}$ & $1.72 \pm 0.49^{\mathrm{a}}$ \\
\hline AST $(\mathrm{IU})$ & $4.667 \pm 1.617$ & $3.600 \pm 1.590$ \\
\hline Clucose $(\mathrm{mg} / \mathrm{dl})$ & $294.65 \pm 69.97^{\mathrm{a}}$ & $257.10 \pm 46.97^{\mathrm{a}}$ \\
\hline Total protein $(\mathrm{g} / \mathrm{dl})$ & $5.97 \pm 1.19$ & $5.71 \pm 1.55$ \\
\hline ALT $(\mathrm{IU})$ & $0.028 \pm 0.017^{\mathrm{a}}$ & $0.006 \pm 0.010^{*}$ \\
\hline Creatinin $(\mathrm{mg} / \mathrm{dl})$ & $0.22 \pm 0.07$ & $0.20 \pm 0.0$ \\
\hline LDH $(\mathrm{mg} / \mathrm{dl})$ & $467.95 \pm 349.21$ & $544.95 \pm 358.01$ \\
\hline
\end{tabular}

Differences between T0 and T3 within the same row $(p<0.05)$.

ALT: alanine aminotransferase; AST: aspartate aminotransferase; LDH: lactate dehydrogenase; SD: standard deviation; TO: before anesthesia; T3: 30 minutes after administration of sevoflurane.

animals by this time point. By $\mathrm{T2}$, the palpebral reflex had completely disappeared in 15 individuals. At T3 there was no sign of this reflex in any of the anesthetized animals. The pedal reflex at $\mathrm{T} 1$ remained strong in 3 chicks, lessened in 14 animals and was completely lost in the other 3 individuals. By T2, no signs of this reflex were observed in 18 chicks. Wing reflex was present in one, lessened in 11, and absent in 8 chickens at T1. By T2, nineteen animals had a decreased wing reflex, that was absent in all animals by T3. Grading of reflex responses is presented in Table 3. The presence of the palpebral reflex, as well as eye opening, were considered to determine recovery time, which lasted between 45 and 270 min (mean= $125.75 \mathrm{~min}$ ). No differences were found for the pedal and palpebral reflexes, or withdrawal of wings when TO and recovery time were compared.

\section{Biochemical findings}

Mean values for ALT, CRE, LDH, ALB, GLU, total protein (TP), and AST at TO and T3 are presented in Table 4. No differences were found between these time points for TP, AST, CRE or LDH. Conversely, albumin, ALT, and GLU were lower at T3, when compared to TO $(p<0.05)$.

\section{Discussion}

The anesthetic protocol used for chickens in this study was safe, with a rapid induction period and a smooth recovery interval. Respiratory rate decreased following 
premedication and remained lower than baseline values (TO) through later stages of anesthesia and the recovery period. However, there were no irreversible complications. This decrease is believed to have derived from an increased level of circulating catecholamines after anesthetic administration. Moreover, a negative effect of medetomidine on the respiratory system has been previously reported in ducks. ${ }^{14,15}$

The mean heart rate of animals during anesthesia in this study was lower than that reported as normal for chicks of over 20 weeks of age (249 \pm 7.0 beats per minute). ${ }^{16}$ There was a sharp descent in mean heart rate after the injection of preanesthetic agents ( $\mathrm{T} 1$ ) that continued throughout the anesthesia period. By reanimation, this value was partially recovered, but remained lower than the normal heart rate in chickens of 250-300 beats per minute. ${ }^{17,18}$ No changes in mean heart rate were found during anesthesia in chickens that were subjected only to a sevoflurane protocol. ${ }^{19}$ However, the heart rate of pigeons, decreased suddenly during pre-anesthesia and continued to descend gradually when anesthetized with medetomidine and ketamine. ${ }^{2}$ Similarly, heart rate was reduced in domestic pigeons and White Leghorn cockerels after medetomidine-ketamine or xylazine-ketamine administration. 20,21 There have been reports of transient hypertension, bradycardia, and apnea, as well as the need of resuscitation maneuvers when medetomidine, midazolam, ketamine and propofol combinations were used in ducks. ${ }^{15}$ However, no critical situations arose with the anesthetic agent combination used in this study.

General ventricular tachycardia was observed in chicks before anesthesia, probably as a consequence of stress due to handling. ${ }^{23}$ Ventricular tachycardia was also observed in some individuals while under anesthesia, probably due to myocardial hypoxia. ${ }^{24}$

When anesthetized, avian species T waves become smaller in the ECG and eventually disappear, whilst $\mathrm{R}$ waves increase and $\mathrm{S}$ waves decrease in magnitude. ${ }^{24}$ The most significant changes observed in the ECG in this study were an increase in both the P wave amplitude and the ST time. Bahri et al. observed an inverse correlation between heart rate and $\mathrm{P}$ wave interval in chickens. ${ }^{17}$ Expansion of $\mathrm{P}$ waves has also been observed in atrial depolarization abnormalities such as hypertrophic myopathies, systemic hypertension, and mitral stenosis. ${ }^{25,26}$ The increase in P wave amplitude found for animals in this work may be associated with dyspnea induced by the anesthetic agents. As for the ST segment, it may often be absent or short in healthy pigeons and parrots. In this study, high ST time values could be an effect of myocardial hypoxia.

One of the most common problems during poultry anesthesia procedures is hypothermia, which increases the recovery period. ${ }^{27}$ The decrease in cloacal body temperature are thought to be related to the vasodilating effects of the anesthetic agents as well as the physiological characteristics of the animals (hypotension, bradycardia and tachypnea). ${ }^{22}$ Nonetheless, the decrease in cloacal temperature that occurred following preanesthetic agent injection in animals in this study, recovered to approach baseline values $\left(39.17 \pm 0.80^{\circ} \mathrm{C}\right)$ by the end of sevoflurane administration (T3).

Similar to results from our work, pigeons exposed to medetomidine, butorphanol, or ketamine have been reported to have good muscle relaxation and adequate analgesia. ${ }^{13,28}$ In the present study, eyes were closed following premedication and the palpebral reflex disappeared after KET and SEVO administration in all chickens. 
Previous studies with caged birds report that the palpebral reflex remains present when the level of anesthesia is mild, and then disappears at moderate or deeper levels. ${ }^{29}$ Recovery periods have been found to span between 126 and 135 minutes in chicks undergoing sevoflurane anaesthesia. ${ }^{30}$ Animals from this study presented longer recovery times (mean 125.75 min, range 45-270 min).

Glucose levels can increase after sevoflurane anesthesia in humans and goats, or isoflurane anesthesia in eagles. 31,32 It has been suggested that this may be related to an inhibition of insulin secretion, or to a high metabolic rate in birds. ${ }^{32,33}$ Levels of glucose were lower at T3 than at TO in animals in this study, which could be attributed to a lower metabolic rate in chickens when compared to wild birds.

Most anesthetic agents are metabolized in the liver and assessment of the ALT enzyme, as well as other biochemical parameters can be used as an indication of hepatic health. ${ }^{4,31}$ In the present study, neither creatinine nor LDH levels were altered but ALT concentrations were lower at T3 when compared to baseline values. These findings were considered to be due to the effects of catecholamines, anesthetic stress, and hepatic metabolism of anesthetic agents.

\section{Conclusion}

Mild changes in glucose levels (hyperglycemia), body temperature, and heart and respiratory rates (tachycardia and dyspnea) were observed after a $\mathrm{MED}+\mathrm{MID}+\mathrm{KET}+\mathrm{SEVO}$ anesthesia protocol in chickens. However, none of these alterations were critical or life-threatening. Therefore, the MED + MID + KET + SEVO combination can be used to obtain satisfactory anesthesia in chickens. Further studies are needed to fully assess the use and qualities of this drug combination for anesthesia protocols in this and other bird species. 


\section{Funding}

This Project was supported by the Department of Scientific Research Projects, Erciyes University, Turkey (Project TYL-2016-6218).

\section{Acknowledgements}

This research was sanctioned by Erciyes University local Ethics Committee Number: 16/006, date: 13.01.2016.

\section{Conflicts of interest}

The authors have no conflicts of interest to declare.

\section{Author contributions}

G. A. designed the study; G. A., Y. C., U. A. and M. K. Y. performed anesthesia procedures; V. G. assessed ECG and biochemical parameters; G. A., Y. C. and U. A. analyzed data and prepared the manuscript.

\section{References}

1. Doneley B. Avian medicine and surgery in practice: companion and aviary birds. Boca Raton, FL: CRC Press; 2016.

2. Machin KL. Avian and exotic pet medicine. London: WB Saunders; 2004.

3. Mohammad FK, Faris GAM, Al-Zubeady AZ. Developmental and behavioral effects of medetomidine following in ovo injection in chicks. Neurotoxicol Teratol. 2012;34:214-8.

4. Grimm KA, Lamont LA, Tranquilli WJ, Greene SA, Robertson S. Veterinary anesthesia and analgesia. Hoboken, NJ: John Wiley \& Sons; 2015.

5. Mohammed FK, Al-Badrany MS, Al-Hasan AM. Detomidine Ketamine anesthesia in chickens. Vet Rec. 1993;133:192.

6. Christensen J, Fosse RT, Halvorsen OJ, Morild I. Comparison of various anesthetic regimens in the domestic fowl. Am J Vet Res. 1987;48(11):1649-57.

7. Pieri L, Schaffner R, Scherschlicht R, Polc P, Sepinwall J, Davidson A, et al. Pharmacology of midazolam. Arzneimittel-Forschung. 1981;31(12a):2180-201.

8. Reves JD, Fragen RJ, Vinik HR, Greenblatt DJ. Midazolam: pharmacology and uses. Anesthesiology. 1985;62(3):310-24.

9. Javdani M, Nikousefat Z, Ghashghaei A, Hashemnia M. Efficacy of ketamine in combination with four common analgesics on some hematological factors of broiler chickens. Int J Livest Res. 2014;4(2):1-9

10. Quandt JE, Greenacre CB. Sevoflurane anesthesia in psittacines. J Zoo Wild Med. 1999;30(2):308-9.

11. Natalini CC. Sevoflurane, desflurane and xenon new inhaled anesthetics in veterinary medicine. Cienc Rural. 2001;31(1):177-83.

12. Naganobu K, Fujisawa Y, Ohde H, Matsuda Y, Sonoda T, Ogawa H. Determination of the minimum anesthetic concentration and cardiovascular dose response for sevoflurane in chickens during controlled ventilation. Vet Surg. 2000;29(1):102-5.

13. Atalan G, Uzun M, Demirkan I, Yıldız S, Cenesiz M. Effects of medetomidine-butorphanol-ketamin anaesthesia and atipamezole on heart and respiratory rate and cloacal temperature of domestic pigeons. J Vet Med A. 2002;49(1):281-5. 
14. Kuenzel WJ, Kusiak JW, Augustine PC, Pitha J. Effect of a beta-adrenergic antagonist on blood pressure, heart rate and beta-adrenoceptors in turkey poults. Comp Biochem Phys C. 1983;76(2):371-5.

15. Machin KL, Caulkett NA. Cardiopulmonary effects of propofol and a medetomidine-midazolam-ketamine combination in mallard ducks. J Vet Anm Res. 1998;59(5):598-602.

16. Sturkie PD, Chillseyzn J. Heart rate changes with age in chickens. Poultry Science. 1972;51(3):906-11.

17. Altimiras J, Crossley DA. Control of blood pressure mediated by baroreflex changes of heart rate in the chicken embryo (Gallus gallus). Am J Physiol Regul Integr Comp Physiol. 2000;278(4):980-6.

18. Bahri E, Nesrin S, Bölükbaşı MF. Tavuklarda Elektrokardiyogram. Lalahan Hayvanclık Araştırma Enstitüsü Dergisi. 1985;25(1):79-86.

19. Naganobu K, Hagio M. Dose-related cardiovascular effects of isoflurane in chickens during controlled ventilation. J Vet Med Sci. 2000;62(4):435-7.

20. Uzun M, Yıldız S, Atalan G, Kaya M, Sulu N. Effects of medetomidine-ketamine combination anesthesia on electrocardiographic findings, body temperature, and heart and respiratory rates in domestic pigeons. Turk J Vet Anim Sci. 2003;27(2):377-82.

21. Maiti SK, Tiwary R, Vasan P, Dutta A. Xylazine, diazepam and midazolam premedicated ketamine anesthesia in White Leghorn cockerels for typhlectomy. J S Afr Vet Assoc. 2006;77(1):12-8.

22. Cornick-Seahorn JL. Veterinary anesthesia. Oxford: Butterworth-Heinemann; 2001.

23. Ritchie BW, Harrison GJ, Harrison LR. Avian medicine: principles and application. Lake Worth: Wingers Publishing; 1994.

24. Miller W, Buttrick M. Current anesthesia recommendations for companion birds. lowa State University Veterinarian. 1999;61(2):3.

25. Brouwers PJ, Wijdicks EF, Hasan D. Serial electrocardiographic recording in aneurysmal subarachnoid hemorrhage. Stroke. 1989;20:1162-7.

26. Cruickshank JM, Neil-Dwyer G, Brice J. Electrocardiographic changes and their prognostic significance in subarachnoid haemorrhage. J Neurol Neurosurg Psychiatry. 1974;37:755-9.

27. Strachan F. Large animal anesthesia. In: Welsh $L$, ed. Anaesthesia for veterinary nurses. 2nd ed. London: Blackwell Publishing; 2009.

28. Lichtenberger $\mathrm{M}, \mathrm{Ko} \mathrm{J}$. Anesthesia and analgesia for small mammals and birds. Vet Clin N Am-Exot. 2007;10(2):293-315.

29. Arnall L. Anaesthesia and surgery in cage and aviary birds. Vet Rec. 1961;73:139-42.

30. Naganobu K, Ise K, Miyamoto T, Hagio M. Sevoflurane anesthesia in chickens during spontaneous and controlled ventilation. Vet Rec. 2003;152(2):45-8.

31. Chan FT, Chang GR, Wang HC, Hsu TH. Anesthesia with isoflurane and sevoflurane in the crested serpent eagle (Spilornis cheela hoya): Minimum anesthetic concentration, physiological effects, hematocrit, plasma chemistry and behavioral effects. J Vet Med Sci. 2013;75(1):1591-600.

32. Hall LW, Clarke KW, Trim CM. Veterinary anaesthesia. 10th ed. London: W.B. Saunders; 2000. 


\section{Original Research}

DOl: http://dx.doi.org/10.22201/fmvz.24486760e.2020.1.620

33. Joyner PH, Jones MP, Ward D, Gompf RE, Zagaya N, Sleeman JM. Induction and recovery characteristics and cardiopulmonary effects of sevoflurane and isoflurane in bald eagles. Am J Vet Res. 2008;69(1):13-22. 